\title{
Análise do controle da esteatose hepática em pacientes após cirurgia bariátrica
}

\author{
Analysis of hepatic steatosis control in patients after bariatric surgery \\ Análisis del control de la esteatosis hepática en pacientes después de una cirugía bariátrica
}

\section{Resumo}

Objetivo: Avaliar a associação entre o controle de esteatose hepática (EH) e o pós-operatório de cirurgia bariátrica em uma instituição privada de Sergipe. Metodologia: Estudo retrospectivo observacional, unicêntrico, com dados coletados por meio de formulário online, previamente autorizado, por 57 pacientes sem distinção de sexo, com obesidade grau III ou grau II associada a comorbidades, submetidos à cirurgia bariátrica pelas técnicas de Gastrectomia Vertical (Sleeve Gástrico) ou Gastroplastia Redutora em Y-de-Roux (Bypass Gástrico) por videolaparoscopia, em uma instituição privada de Aracaju/SE, entre 2019 e 2021. Após aplicados os critérios de exclusão, foram analisados 45 pacientes. Dados analisados por meio de frequência percentual, testes de Pearson e de McNemar. Nível de significância adotado de 5\%. Resultado: No pré-operatório foi encontrada prevalência de EH em $66,7 \%$ desses e no pós-operatório a prevalência encontrada foi de $16,7 \%$, com redução do grau da patologia ao exame ultrassonográfico para EH grau I. A cirurgia bariátrica foi curativa em 83,3\% dos pacientes com EH no pré-operatório, possuindo melhora clínica em 23 (67,6\%) daqueles submetidos ao Sleeve Gástrico e 7 (63,6\%), ao Bypass Gástrico. Conclusão: Percebeu-se associação significativa entre cirurgia bariátrica e a resolução da esteatose hepática, independente da técnica cirúrgica utilizada.

Palavras-chave: Esteatose hepática; Cirurgia bariátrica; Controle.

\begin{abstract}
Purpose: To evaluate the association between the control of hepatic steatosis (HS) and the postoperative period of bariatric surgery in a private institution in Sergipe. Methodology: Retrospective observational and single-center study, with data collected through an online form, previously authorized by 57 patients, regardless of gender, with Class III or Class II obesity associated with comorbidities, who underwent bariatric surgery using the techniques of Vertical Gastrectomy (Gastric Sleeve) and Roux-en-Y Reduction Gastroplasty (Gastric Bypass) by videolaparoscopy, in a private institution in Aracaju/SE, between 2019 and 2021. After applying the exclusion criteria, 45 patients were analyzed. Data were analyzed using percentage frequency, Pearson and McNemar tests. It was adopted a significance level of 5\%. Results: In the preoperative period, it was found a $66.7 \%$ prevalence of HS and in the postoperative period the prevalence was found to be $16.7 \%$, with a reduction in the degree of the pathology to HS class I, verified through ultrasonographic examination. There was an $83.3 \%$ success rate in the patients with HS in the preoperative period. Of the $83.3 \%$, there was a clinical improvement in $23(67.6 \%)$ of those who underwent Gastric Sleeve surgery, as well as an improvement of 7 (63.6\%) who underwent Gastric Bypass surgery. Conclusion: There was a significant association between bariatric surgery and the resolution of hepatic steatosis, regardless of the surgical technique used.
\end{abstract}

Keywords: Hepatic steatosis; Bariatric surgery; Control.

\section{Resumen}

Finalidad: Evaluar la asociación entre el control de la esteatosis hepática (EH) y el período postoperatorio de cirugía bariátrica en una institución privada en Sergipe. Metodología: Estudio observacional retrospectivo y unicéntrico, con datos recopilados a través de un formulario en línea, previamente autorizados por 57 pacientes, independientemente del 
género, con obesidad de Clase III o Clase II asociada con comorbilidades, que se sometieron a cirugía bariátrica utilizando las técnicas de Gastrectomía Vertical (Manga Gástrica) y Gastroplastia de Reducción Roux-en-Y (Bypass Gástrico) por videolaparoscopia, en una institución privada en Aracaju/SE, entre 2019 y 2021. Después de aplicar los criterios de exclusión, se analizaron 45 pacientes. Los datos se analizaron utilizando porcentaje de frecuencia, pruebas de Pearson y McNemar. Se adoptó un nivel de significación del 5\%. Resultados: En el período preoperatorio, se encontró una prevalencia del 66,7\% de HS y en el período postoperatorio se encontró que la prevalencia era del 16,7\%, con una reducción en el grado de patología a HS clase I, verificada a través de un examen ultrasonográfico. Hubo una tasa de éxito del 83,3\% en los pacientes con HS en el período preoperatorio. Del 83,3\%, hubo una mejora clínica en 23 (67,6\%) de los que se sometieron a cirugía de manga gástrica, así como una mejora de 7 (63,6\%) que se sometieron a cirugía de bypass gástrico. Conclusión: Hubo asociación significativa entre la cirugía bariátrica y la resolución de la esteatosis hepática, independientemente de la técnica quirúrgica utilizada.

Palabras clave: Esteatosis hepática; Cirugía bariátrica; Control.

\section{Introdução}

A esteatose hepática relacionada à obesidade abrange disfunções hepáticas que variam da esteatose não alcoólica e, por conseguinte, fibrose hepática e cirrose (Wirth et al., 2020). A concentração de lipídios nos hepatócitos caracteriza a esteatose hepática (EH), sendo provavelmente a principal causa de morbimortalidade relacionada a patologias hepáticas. Há também a presença de uma relação direta entre resistência insulínica, gordura visceral e fatores de risco de fonte metabólica e cardiovasculares com elevada prevalência da obesidade (Frare, Alves, Farias, Medeiros, \& Soares, 2021; Soler et al, 2008).

A ultrassonografia abdominal é o exame de imagem de primeira linha largamente utilizado para o diagnóstico dessa doença. A prevalência da $\mathrm{EH}$ em pacientes com obesidade de forma grave que realizaram a cirurgia bariátrica pode ultrapassar os 90\% (Beymer et al., 2003; Goldoni et al., 2020). Naqueles com doenças associadas à obesidade, a melhor escolha terapêutica para a perda de peso e redução das comorbidades é a cirurgia bariátrica, com resultados positivos no controle da maior parte das patologias para pacientes refratários ao tratamento clínico conservador (Castanha et al., 2018; Hachem, \& Brennan, 2016; Meré, \& Blanco, 2004; Vargas et al., 2012).

Dentre as técnicas cirúrgicas utilizadas, o bypass em Y-de-Roux (bypass gástrico) e a gastrectomia vertical (sleeve gástrico) são as analisadas nos estudos. Entretanto, ainda não está esclarecido qual técnica está relacionada aos benefícios sobre a EH (Billeter et al., 2016; Froylich et al., 2016; Goldoni et al., 2020; Raj et al., 2015). Esses procedimentos cirúrgicos agem diretamente no controle da dislipidemia e na redução da resistência insulínica, presumindo-se, portanto, que interfiram na regressão da EH (Bult, van Dalen., \& Muller, 2008; Hafeez, \& Ahmed, 2013; Junior, Nascimento, Nazário, \& Mick, 2015). O estudo tem como objetivo avaliar a associação entre o controle de EH e o pós-operatório de cirurgia bariátrica dos pacientes em uma instituição privada de Sergipe, por meio da comparação de exames ultrassonográficos pré e pós-operatórios desses pacientes.

\section{Metodologia}

Trata-se de um estudo retrospectivo observacional, unicêntrico, realizado a partir da coleta de dados por meio de contato telefônico com os participantes e, posteriormente, envio de formulário online, previamente autorizado, para pacientes submetidos à cirurgia bariátrica pelas técnicas de Gastrectomia Vertical (Sleeve) e Gastroplastia Redutora em Y-de-Roux (Bypass Gástrico) por videolaparoscopia, em uma instituição privada de Aracaju/SE, entre 2019 e 2021 (Estrela, 2018). Os dados analisados foram idade, gênero, presença de EH antes e após cirurgia bariátrica, além da técnica cirúrgica utilizada. Foram incluídos pacientes que foram submetidos a cirurgias bariátricas eletivas, sem distinção de sexo, com obesidade grau III ou grau II associada a comorbidades. Os critérios de exclusão foram os pacientes submetidos a outros procedimentos cirúrgicos que não os supracitados, aqueles que não sabiam informar o grau de EH e os que perderam seguimento clínico da patologia no pós-operatório.

As variáveis categóricas foram descritas por meio de frequência absoluta e relativa percentual. As variáveis contínuas foram descritas por meio de média e desvio padrão. A hipótese de independência entre variáveis categóricas foi testada por meio 
do teste Qui-Quadrado de Pearson. A hipótese de igualdade de proporções pareadas foi testada por meio do teste de McNemar. O nível de significância adotado foi de 5\% e o software utilizado foi o R Core Team 2021 (versão 4.1.0). Este estudo foi submetido e aprovado pelo Comitê de Ética em Pesquisa (CEP) através da Plataforma Brasil, sob o parecer no 4.812.212. As informações foram aceitas através da assinatura do Termo de Consentimento Livre e Esclarecido conforme as normas do Conselho Nacional de Saúde do Ministério da Saúde explicitadas na resolução 466/20122.

\section{Resultados}

Durante o período analisado, 2019 a 2021, em uma instituição privada de Aracaju/SE, 57 pacientes foram submetidos à cirurgia bariátrica, entretanto 12 foram excluídos por não apresentarem seguimento clínico e ultrassonográfico após a cirurgia, restando, então, 45 pacientes no estudo. A idade mínima foi 22 anos e a máxima, 60 anos, constituindo a média de idade de 37,0 \pm 9,5 anos, com predominância do sexo feminino (Tabela 1).

Tabela 1: Perfil epidemiológico de pacientes submetidos à cirurgia bariátrica.

\begin{tabular}{lllll}
\hline & $\mathrm{n}$ & $\%$ & Média & $\mathrm{DP}$ \\
\hline Idade & & & 37,0 & 9,5 \\
Sexo & & & \\
Feminino & 31 & 68,9 \\
Masculino & 14 & 31,1 \\
\hline \multicolumn{4}{l}{} \\
Legenda: n - frequência absoluta. \% - \\
frequência relativa percentual. DP - Desvio \\
padrão. \\
Fonte: Dados da pesquisa (2021).
\end{tabular}

Um quantitativo maior de pacientes foi submetido ao Sleeve Gástrico $(75,6 \%)$ em detrimento do Bypass Gástrico $(24,4 \%)$ (Tabela 2).

Tabela 2: Prevalência da técnica cirúrgica bariátrica utilizada.

\begin{tabular}{lcccc}
\hline & $\mathrm{n}$ & $\%$ & Média & $\mathrm{DP}$ \\
\hline Técnica cirúrgica & & & & \\
Sleeve Gástrico & 34 & 75,6 & & \\
Bypass Gástrico & 11 & 24,4 & & \\
\hline
\end{tabular}

Legenda: $\mathrm{n}$ - frequência absoluta. \% - frequência relativa percentual. DP - Desvio padrão.

Fonte: Dados da pesquisa (2021).

Além disso, foi analisada a prevalência da EH anteriormente à bariátrica com a distribuição do grau dessa patologia. Do total de pacientes investigados, $30(66,7 \%)$ apresentavam $\mathrm{EH}$ ao exame ultrassonográfico pré-operatório e 15 (33,3\%) não possuíam tal comorbidade (Tabela 3). Os graus mais encontrados foram de EH grau II e III presentes em 11 (39,3\%) pacientes em cada grau. No pós-operatório, a maior parte dos pacientes apresentaram-se sem EH (88,9\%) e apenas 11,1\% apresentavam EH, porém com redução para EH grau I (Tabela 3). 
Tabela 3: Prevalência e distribuição em grau da esteatose hepática pré e pós cirurgia bariátrica.

\begin{tabular}{lllll}
\hline & $\mathrm{n}$ & $\%$ & Média & $\mathrm{DP}$ \\
\hline EH pré cirurgia bariátrica & & & & \\
Sim & 30 & 66,7 & & \\
Não & 15 & 33,3 & & \\
Grau & & & & \\
I & 8 & 21,4 & & \\
II & 11 & 39,3 & & \\
III & 11 & 39,3 & &
\end{tabular}

EH pós cirurgia bariátrica

\begin{tabular}{lll} 
Sim & 5 & 11,1 \\
Não & 40 & 88,9 \\
Grau & & \\
I & 5 & 100 \\
II & 0 & 0 \\
III & 0 & 0 \\
\hline
\end{tabular}

Legenda: $\mathrm{EH}$ - esteatose hepática. $\mathrm{n}$ - frequência absoluta. \% - frequência relativa percentual. DP - Desvio padrão.

Fonte: Dados da pesquisa (2021).

No pós-operatório, foi acompanhada a evolução clínica dos 30 pacientes que apresentavam EH anteriormente. (Tabela 4). Desses, 67,6\% haviam sido submetidos ao Sleeve e 63,6\%, ao Bypass gástrico (Tabela 5).

Tabela 4: Evolução clínica da amostra avaliada.

\begin{tabular}{|c|c|c|c|}
\hline & $\mathrm{n}$ & $\%$ & Média DP \\
\hline \multicolumn{4}{|l|}{ Evolução } \\
\hline Evoluiu & 30 & 66,7 & \\
\hline Não tinha EH & 15 & 33,3 & \\
\hline \multicolumn{4}{|c|}{$\begin{array}{l}\text { Legenda: } \mathrm{EH}-\text { esteatose hepática. } \mathrm{n}- \\
\text { frequência absoluta. } \% \text { - frequência relativa } \\
\text { percentual. DP - Desvio padrão. } \\
\text { Fonte: Dados da pesquisa }(2021) \text {. }\end{array}$} \\
\hline
\end{tabular}

Tabela 5: Relação entre a técnica cirúrgica realizada e a evolução clínica da esteatose hepática em pacientes submetidos à cirurgia bariátrica.

\begin{tabular}{llll}
\hline & \multicolumn{2}{c}{ Técnica Cirúrgica } & \\
\cline { 2 - 3 } & $\begin{array}{l}\text { Sleeve Gástrico } \\
\mathrm{n}(\%)\end{array}$ & $\begin{array}{l}\text { Bypass Gástrico } \\
\mathrm{n}(\%)\end{array}$ & p-valor \\
\hline $\begin{array}{l}\text { Evolução } \\
\begin{array}{l}\text { Evoluiu } \\
\text { Não tinha EH }\end{array}\end{array}$ & $23(67,6)$ & $7(63,6)$ & 1,000 \\
\hline $\begin{array}{l}\text { Legenda: EH - esteatose hepática. } \\
\text { frequência relativa percentual. Teste Qui-Quadrado. }\end{array}$ & \\
Fonte: Dados da pesquisa (2021).
\end{tabular}

Quanto à prevalência da EH, constata-se uma elevada remissão dessa patologia após a cirurgia bariátrica, com 83,3\% de cura dos pacientes dessa amostra (Tabela 6). Em contrapartida, apenas 16,7\% (Tabela 6) dos que apresentavam EH no pré- 
operatório permaneceram com a doença, entretanto, com redução significativa da EH ao exame ultrassonográfico para EH grau I, desde que o valor de p encontrado foi menor que 0,001 (Tabela 7).

Tabela 6: Comparação entre a frequência dos pacientes com EH pré e pós cirurgia bariátrica.

\begin{tabular}{|c|c|c|c|}
\hline & \multicolumn{2}{|c|}{ EH pré cirurgia bariátrica } & \multirow[b]{2}{*}{ p-valor } \\
\hline & $\begin{array}{l}\text { Sim } \\
\mathrm{n}(\%)\end{array}$ & $\begin{array}{l}\text { Não } \\
\text { n (\%) }\end{array}$ & \\
\hline \multicolumn{4}{|c|}{ EH pós C.B. } \\
\hline Sim & $5(16,7)$ & $0(0,0)$ & $<0,001$ \\
\hline Não & $25(83,3)$ & $15(100)$ & \\
\hline
\end{tabular}

Ao correlacionar a distribuição do graus definida pelo achado ultrassonográfico de EH em pacientes submetidos a cirurgia bariátrica, foi averiguado que dos 8 pacientes que apresentavam EH grau I, todos obtiveram remissão. Dos 11 pacientes com EH grau II anteriormente à cirurgia, 90,9\% obtiveram remissão. Ao analisar os 11 pacientes com EH grau III no préoperatório, observa-se que $63,6 \%$ não apresentaram a doença no pós-operatório (Tabela 7).

Tabela 7: Distribuição comparativa entre pacientes submetidos a cirurgia bariátrica com EH pré e pós-operação.

\begin{tabular}{|c|c|c|c|}
\hline & \multicolumn{2}{|c|}{ EH pós cirurgia bariátrica } & \multirow[b]{2}{*}{ p-valor } \\
\hline & $\begin{array}{l}\text { Não } \\
\mathrm{n}(\%)\end{array}$ & $\begin{array}{l}\text { Sim, Grau I } \\
\mathrm{n}(\%)\end{array}$ & \\
\hline \multicolumn{4}{|c|}{ EH pré C.B } \\
\hline Não & $15(100)$ & $0(0)$ & 0,039 \\
\hline Grau I & $8(100)$ & $0(0)$ & \\
\hline Grau II & $10(90,9)$ & $1(9,1)$ & \\
\hline Grau III & $7(63,6)$ & $4(36,4)$ & \\
\hline
\end{tabular}

\section{Discussão}

A obesidade é considerada o fator de risco mais importante no desenvolvimento de doença hepática não-alcoólica (DHGNA) (Freitas, Freitas, Parolin, Campos, \& Coelho, 2007). Segundo Freitas et al. (2007), diversos estudos demonstram a prevalência de EH em pacientes com indicação de cirurgia bariátrica, assim como uma pesquisa que apontou prevalência de tal comorbidade em pacientes com obesidade de 76\% (Bellentani et al., 2000) e outro estudo que demonstrou prevalência de $86 \%$ para esteatose em 551 pacientes obesos (Marceau et al., 1999). No presente estudo 66,7\% pacientes submetidos à cirurgia bariátrica apresentavam EH no pré-operatório, sendo 39,3\% deles grau III (esteatose grave), 39,3\% de grau II (esteatose moderada) e $21,4 \%$ de grau I (esteatose leve).

Diversas metanálises evidenciam que a esteatose hepática tem melhora ou resolução completa na maioria dos pacientes após a cirurgia bariátrica (Fakhry et al., 2019), assim como foi demonstrado por Mummadi, Kasturi, Chennareddygari, \& Sood 
(2008), em que 91,6\% dos pacientes exibiram resultados favoráveis, e por Bower et al. (2015), que evidenciaram uma melhora em 50,2\% dos pacientes após a bariátrica. Nesse estudo, 83,3\% dos pacientes com EH apresentaram remissão após a realização da cirurgia bariátrica. Além disso, foi demonstrado que apenas 16,7\% mantiveram a EH posteriormente ao procedimento cirúrgico, porém com melhora no grau de acometimento, com redução para EH grau I. Tais dados demonstram com significância estatística que a cirurgia bariátrica concede uma remissão direta da esteatose hepática na maioria dos pacientes submetidos ao procedimento.

De acordo com a literatura, há uma evidente melhora da EH após realização de procedimentos bariátricos, contudo não parece haver diferença estatística entre os resultados encontrados nas técnicas Sleeve e Bypass. Alguns estudos como os publicados por Froylich et al. (2016), Billeter et al. (2016) e Raj et al. (2015), explanaram sobre os resultados pós-operatórios comparando as técnicas supracitadas e demonstraram não encontrar diferenças estatísticas entre essas técnicas. Todavia, Goldoni et al. (2021) afirmam em seu estudo que o Bypass apresentou melhores resultados estatísticos. No atual estudo, os percentuais de melhora da EH após Sleeve e após Bypass apresentaram resultados semelhantes. Assim, a diferença estatística entre as técnicas estudadas foi insignificante, o que evidencia a necessidade de mais pesquisas de longo prazo sobre os impactos específicos de ambos os procedimentos de cirurgia bariátrica sobre a esteatose hepática.

\section{Conclusão}

A cirurgia bariátrica é um procedimento eficaz para promover o controle da evolução da esteatose hepática, seja com a melhora ou com a remissão completa da doença, independente da técnica cirúrgica utilizada. Houve associação estatisticamente significativa entre cirurgia bariátrica e a resolução da comorbidade estudada. Entretanto, uma possível limitação do estudo é o tamanho da amostra, por se tratar de uma base de dados com pequeno quantitativo de pacientes. Esse fato confirma a necessidade de estabelecimento de ensaios clínicos bem desenhados e de longo prazo, com amplo banco de dados para melhor entendimento dos benefícios da cirurgia bariátrica no controle da esteatose hepática.

\section{Referências}

Bellentani, S., Saccoccio, G., Masutti, F., Crocè, L. S., Brandi, G., Sasso, F., Cristanini, G, \& Tiribelli, C. (2000). Prevalence of and risk factors for hepatic steatosis in Northern Italy. Annals of internal medicine, 132(2), 112-117. 10.7326/0003-4819-132-2-200001180-00004

Beymer, C., Kowdley, K. V., Larson A., Edmonson, P., Dellinger, E. P., \& Flum, D. R. (2003). Prevalence and predictors of asymptomatic liver disease in patients undergoing gastric Bypass surgery. Arch Surg, 138(111), 1240-1244. 10.1001/archsurg.138.11.1240

Billeter, A. T., Senft, J., Gotthardt, D., Knefeli, P., Nickel, F., Schulte, T., Fischer, L., Nawroth, P. P., Büchler, M.W., \& Müller-Stich, B. P. (2016). Combined non-alcoholic fatty liver disease and type 2 diabetes mellitus: sleeve gastrectomy or gastric bypass?-a controlled matched pair study of 34 patients. Obesity surgery, 26(8), 1867-1874. 10.1007/s11695-015-2006-y

Bower, G., Toma, T., Harling, L., Jiao, L. R., Efthimiou, E., Darzi, A., Athanasiou, T., \& Ashrafian, H. (2015). Bariatric surgery and non-alcoholic fatty liver disease: a systematic review of liver biochemistry and histology. Obesity surgery, 25(12), 2280-2289. 10.1007/s11695-015-1691-x

Bult, M. J., van Dalen, T., \& Muller, A. F. (2008). Surgical treatment of obesity. European journal of endocrinology, 158(2), 135-145. 10.1530/EJE-07-0145

Castanha, C. R., Ferraz, A. A. B., Castanha, A. R., Belo, G. D. Q. M. B., Lacerda, R. M. R., \& Vilar, L. (2018). Avaliação da qualidade de vida, perda de peso e comorbidades de pacientes submetidos à cirurgia bariátrica. Revista do Colégio Brasileiro de Cirurgióes, 45, e-20181864. 10.1590/0100-6991e-20181864

Fakhry, T. K., Mhaskar, R., Schwitalla, T., Muradova, E., Gonzalvo, J. P., \& Murr, M. M. (2019). Bariatric surgery improves nonalcoholic fatty liver disease: a contemporary systematic review and meta-analysis. Surgery for Obesity and Related Diseases, 15(3), 502-511. 10.1016/j.soard.2018.12.002

Frare, L., Pantano Alves, D., Farias, I. R.., Medeiros, G. M. de, \& Soares, C. F. de P. (2021). Associação de esteatose hepática em pacientes em pré-operatório de cirurgia bariátrica com apneia obstrutiva do sono. Research, Society and Development, 10(6), e36610615495. 10.33448/rsd-v10i6.15495

Freire, M; \& Pattussi, M. (2018). Tipos de estudo In Estrela, C. (Org.), Metodologia Científica: Ciência, Ensino, Pesquisa (pp. 187-188). Editora Artes Médicas.

Freitas, A. C. T. D., Freitas, D. T. D., Parolin, M. B., Campos, A. C. L., \& Coelho, J. C. U. (2007). Doença hepática não-alcoólica: evolução após derivação gastrojejunal em Y-de-Roux pela técnica de fobi-capella. Arquivos de Gastroenterologia, 44, 49-53. 10.1590/S0004-28032007000100011 
Froylich, D., Corcelles, R., Daigle, C., Boules, M., Brethauer, S., \& Schauer, P. (2016). Effect of Roux-en-Y gastric bypass and sleeve gastrectomy on nonalcoholic fatty liver disease: a comparative study. Surgery for Obesity and Related Diseases, 12(1), 127-131. 10.1016/j.soard.2015.04.004

Goldoni, M. B., Fontes, P. R. O., Guimarães, M. M., Diedrich-Neto, J. A., Nogueira, T., Teixeira, U. F., Giacomazzi, C. B., Kiss, G., Pioner, S. R., \& Rodrigues, P. D. (2021). Bypass vs. Sleeve e seus resultados na doença hepática gordurosa não alcoólica: qual a melhor técnica? ABCD. Arquivos Brasileiros de Cirurgia Digestiva (São Paulo), 33. 10.1590/0102-672020200003e1549

Hachem, A, \& Brennan, L. (2016). Quality of life outcomes of bariatric surgery: a systematic review. Obes Surg., 26(2), 395-409. 10.1007/s11695-015-1940-z

Hafeez, S., \& Ahmed, M. H. (2013). Bariatric surgery as potential treatment for nonalcoholic fatty liver disease: a future treatment by choice or by chance? Journal of obesity, 2013, 839275. 10.1155/2013/839275

Junior, L. C. K., do Nascimento, R. R., Nazário, R. F., \& Mick, H. (2015). Estudo ultrassonográfico da esteatose hepática no pré e pós-operatório de cirurgia bariátrica. Revista da AMRIGS, 59(3), 209-216. https://pesquisa.bvsalud.org/portal/resource/pt/biblio-835414

Marceau, P., Biron, S., Hould, F. S., Marceau, S., Simard, S., Thung, S. N., \& Kral, J. G. (1999). Liver pathology and the metabolic syndrome X in severe obesity. The Journal of Clinical Endocrinology \& Metabolism, 84(5), 1513-1517. 10.1210/jcem.84.5.5661

Meré, L. A. F., \& Blanco, M. A. (2004). Obesidad y cirugía bariátrica: Implicaciones anestésicas. NutrHosp, 19(1), 3344. https://scielo.isciii.es/scielo.php?script=sci_arttext\&pid=S0212-16112004000100007

Mummadi, R. R., Kasturi, K. S., Chennareddygari, S., \& Sood, G. K. (2008). Effect of bariatric surgery on nonalcoholic fatty liver disease: systematic review and meta-analysis. Clinical Gastroenterology and Hepatology, 6(12), 1396-1402. 10.1016/j.cgh.2008.08.012

Raj, P. P., Gomes, R. M., Kumar, S., Senthilnathan, P., Karthikeyan, P., Shankar, A., \& Palanivelu, C. (2015). The effect of surgically induced weight loss on nonalcoholic fatty liver disease in morbidly obese Indians:"NASHOST" prospective observational trial. Surgery for Obesity and Related Diseases, 11(6), 13151322. 10.1016/j.soard.2015.02.006

Soler, G. L. N., Silva, A. W. S. M., Silva, V. C. G., \& Teixeira, R. de J. (2008). Doença Hepática Gordurosa Não-Alcoólica: associação com síndrome metabólica e fatores de risco cardiovascular. Rev SOCERJ, 21(2), 94-100. http://sociedades.cardiol.br/socerj/revista/2008_02/a2008_v21_n02_a04Gisele.pdf

Vargas, V., Allende, H., Lecube, A., Salcedo, M. T., Baena-Fustegueras, J. A., Fort, J. M., Rivero, J., Ferrer, R., Catalán, R., Pardina, E., Ramón Y Cajal, S., Guardia, J., \& Peinado-Onsurbe, J. (2012). Surgically induced weight loss by gastric bypass improves non alcoholic fatty liver disease in morbid obese patients. World journal of hepatology, 4(12), 382-388. 10.4254/wjh.v4.i12.382

Wirth, K. M., Sheka, A. C., Kizy, S., Irey, R., Benner, A., Sieger, G., Simon, G., Ma, S., Lake, J., Aliferis, C., Leslie, D., Marmor, S., \& Ikramuddin, S. (2020). Bariatric Surgery is Associated With Decreased Progression of Nonalcoholic Fatty Liver Disease to Cirrhosis: A Retrospective Cohort Analysis. Annals of surgery, 272(1), 32-39. 10.1097/SLA.0000000000003871 